\title{
Graphene electrochemical sensors for the detection of S 29:1-(2-chloro-2-(4-chlorophenyl)ethyl)-N-(4- fluorobenzyl)-1H-pyrazolo[3,4-d]pyrimidin-4-amine: an anticancer drug
}

\author{
Federica Valentini ${ }^{1,2 *}$, Simonetta Antonaroli ${ }^{1}$, Giulia Iovenitti ${ }^{3}$, Maurizio Botta ${ }^{3}$ and Maurizio Talamo ${ }^{2}$ \\ ${ }^{1}$ Science and Chemical Technologies Department, Tor Vergata University, via della Ricerca Scientifica 1, 00133 Rome, Italy \\ ${ }^{2}$ INUIT Foundation Tor Vergata University, via dell'Archiginnasio snc, 00133 Rome, Italy \\ ${ }^{3}$ Biotechnologies, Chemistry and Pharmacy Dep., Siena University, Via Aldo Moro 2, 53100 Siena, Italy
}

\section{Commentary}

Graphene (G) and Graphene derivatives (Gds) represent the next generation of smart materials, having exciting features to apply in Medicine field. Especially, several studies [1,2] report about the biocompatibility of graphene and graphene derivatives in several different cell lines. Biocompatibility for G and Gds seems to be related to main aspects, as: the metal-free chemical composition of graphene and the nano sheets shape. Recently, Valentini, et al. [3] report about the in vitro biocompatibility of Graphene Oxide (GO) nanosheets, tested in different human cell lines and compared with that exhibited by Single Wall Carbon Nanotubes (SWCNTs).

This GO nanomaterial was obtained during 2015 in Tor Vergata University by the Patent N102015000023739, applying the electrochemical exfoliation of highly oriented pyrolitic graphite anode electrodes, in electrolytic working medium. The chemical-physical features and also the biocompatibility of GO sheets, synthesized in this work, were widely reported in our previous paper [3]. Furthermore, antibacterial properties were studied, in presence of Staphylococcus Aureus and Escherichia Coli bacteria, as reported in our previous paper [4].

In this paper, the electrochemical behavior of graphene was investigated in presence of 1-(2-chloro-2-(4-chlorophenyl)ethyl)-N(4-fluorobenzyl)-1H-pyrazolo [3,4-d] pyrimidin-4-amine (labeled as S 29 and, see Figure 1), for the first time. The scientific interest toward this therapeutics was mainly due to the inhibitor role toward the sarcoma ( $\mathrm{Src}$ ) related protein kinase, especially in neuroblastoma, medulloblastoma and osteosarcoma cancer cells [5-7].

For this purpose, the great possibility to assemble an electrochemical portable sensor, able to quantitatively detect S29, during the inhibitor processes in neuroblastoma cell lines, seems to be an extraordinary challenge, in the anticancer drug research fields. According to this consideration, the aim of this study is to assemble and then characterize new miniaturized graphene modified Screen Printed Electrodes (SPEs) system, combined with an electrochemical detection for the quantification of S29 therapeutics. This latter was kindly provided by Prof. M. Botta, and here, a short description of the S29 synthesis was also provided. The synthetic approach to prepare the compound S29 [8] was started from the same reaction between ethylethoxymethylene-

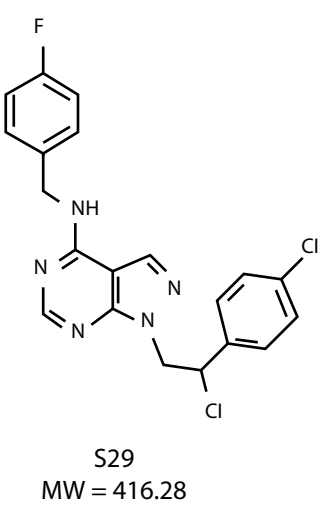

Figure 1. S 29 structure: 1-(2-chloro-2-(4-chlorophenyl)ethyl)-N-(4-fluorobenzyl)-1Hpyrazolo[3,4-d]pyrimidin-4-amine

cyanoacetate [2] and the appropriate 4-fluorophenylhydrazinylethanol [8]. The pyrazole intermediate [9] was reacted with formamide for 8 hours at $190{ }^{\circ} \mathrm{C}$ to give the corresponding pyrazolo[3,4-d] pyrimidinones [10] in high yield. Treatment of the latter with an excess of the Vilsmeier complex (POCl3 /DMF) for 12 hours at reflux

${ }^{\star}$ Correspondence to: Valentini F, Science and Chemical Technologies Department, Tor Vergata University, via della Ricerca Scientifica 1, 00133 Rome, Italy, Tel: (+39)0672594889; Fax: (+39)0672594328; E-mail: federica. valentini@uniroma2.it

Special Issue: Nanotechnology: Challenges and Perspectives in Medicine

Dr. Federica Valentini

Department of Sciences and Chemical Technologies

Tor Vergata University

Italy

Maurizio Talamo

Professor

Department of Enterprise Engineering Italy

Received: September 20, 2018; Accepted: October 08, 2018; Published: October 11,2018 
Valentini F (2018) Graphene electrochemical sensors for the detection of S 29:1-(2-chloro-2-(4-chlorophenyl)ethyl)-N-(4-fluorobenzyl)-1H-pyrazolo[3,4-d] pyrimidin-4-amine: an anticancer drug

in $\mathrm{CHCl} 3$ led to the formation of the halogenated derivative [11]. The C4 chlorine atom was in turn substituted with (4-fluorophenyl) methanamine, in anhydrous toluene at room temperature, to afford the final compound in yields of $75 \%$.

The electrochemical profile of S29 was investigated by applying GO modified Screen Printed Electrodes (GO/SPEs). SPEs are commercially available (purchased by Metrohm DropSens, Origgio (VA) Italy) and then, they are modified by drop casting $6 \mu \mathrm{L}$ of GO nano dispersion, with a concentration of $1 \mathrm{mg} / \mathrm{mL}$, in ethanol. The electrochemical characterization study was performed using these modified GO Screen Printed Electrodes, which correspond to the electrochemical cell, incorporating three electrodes in the same system: a GO based working electrode (3-mm diameter), a $\mathrm{Ag} / \mathrm{AgCl}$ reference electrode, and a carbon pseudo-counter electrode. An Autolab electrochemical system (Eco Chemie, Utrecht, The Netherlands) equipped with PGSTAT-12 and GPES software (Eco Chemie, Utrecht, The Netherlands) was applied for the electrochemical investigation of the electron-transfer properties, exhibited by S29 on GO/SPEs, mainly by using Differential Pulse Voltammetry (DPV) techniques. The electrochemical oxidation profile for S29 was reported on Figure 2A, with am oxidation peak centered around $+400 \mathrm{mV}$, versus $\mathrm{Ag} / \mathrm{AgCl}$ reference electrode. The corresponding calibration curve was also carried out and reported on Figure $2 \mathrm{~B}$ and $\mathrm{C}$. The electrochemical performances of the amperometric sensor seem to be relevant, showing a wide range of concentration $(10-10000 \mathrm{nmol} / \mathrm{L})$, a low detection of limit $(5 \mathrm{nmol} / \mathrm{L})$, a high sensitivity $\left(0.07 \mu \mathrm{Acm}^{-2} \mathrm{nM}^{-1}\right)$, good reproducibility $(-1 \%)$ and short response time (4s). This sensor resulted very innovative because in literature, there is only one scientific paper [9], where Martic, et al. reported on an electrochemical biosensor developed for the determination of sarcoma (Src)-related protein kinase, in presence of pyrimidine-based small molecules, as: pyrazolopyrimidines 4-amino-5-(4-methylphenyl)-7-(tert-butyl)pyrazolo pyrimidine (PP1), 4-amino-5-(4-chlorophenyl)-7-(tert-butyl)pyrazolo pyridine (PP2), which are the standard inhibitors for kinases belonging to the Src family of protein; and the inhibitor of Src protein kinase, 2-oxo3-(4,5,6,7-tetrahydro-1-H-indol-2-ylmethylene)-2,3-dihydro- $1 \mathrm{H}$ indole-5-sulfonic acid dimethylamide (SU6656). The electrochemical principle of this biosensor was completely different than the GO/SPEs, presented in this study and in any case, the analytical performances of the biosensors, prepared by Martic, et al. [9] were not comparable with those observed in this paper (working with GO/SPE systems). Indeed, the linear range of concentration $(5-30 \mathrm{nmol} / \mathrm{L})$ resulted much more limited, working with the inhibition based electrochemical biosensors; with even worse reproducibility (RSD\% 3-5) of the measurements and a longer response time $(20 \mathrm{~min})$.

In addition, the electrochemical GO/SPEs were highly selective sensors, mainly toward one of several isomeric forms of pyrazolopyrimidines, as the pyrazolo[3,4-d] pyrimidine (labeled as SI 306) (Figure 3). This latter was also kindly provided by Prof M. Botta (Siena University) and synthesised according to the literature [10]. The high selectivity, exhibited by the GO/SPE devices, certainly could represent a future perspective in Medicine, providing the great opportunity to specifically quantify both anticancer drugs (as S29 and Si306).

In conclusion, in this work GO was electrochemically synthesised and fully characterized, for the assembly of modified SPEs, applied for the quantification of S29 therapeutic molecules. Analytical results showed a wide range of concentration, a low detection of limit, good sensitivity, high reproducibility and very short response time. This

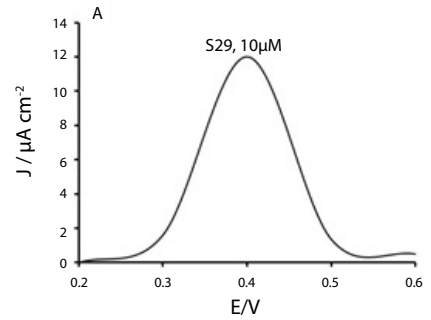

A

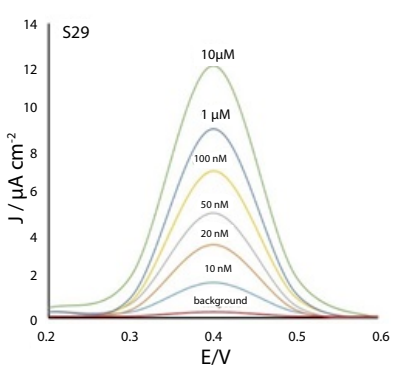

B

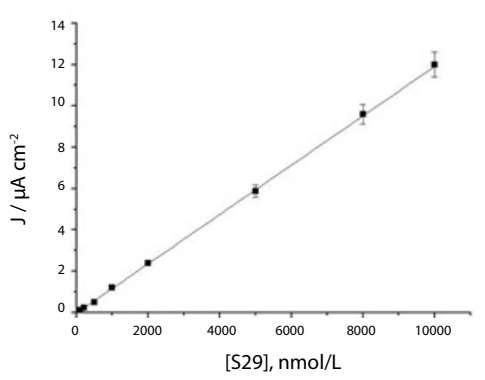

Figure 2. (A): The typical oxidation peak for S 29 drug, recorded by DPV; (B): Calibration plot for S 29, carried out by DPV techniques; and (C) the linear regression plot

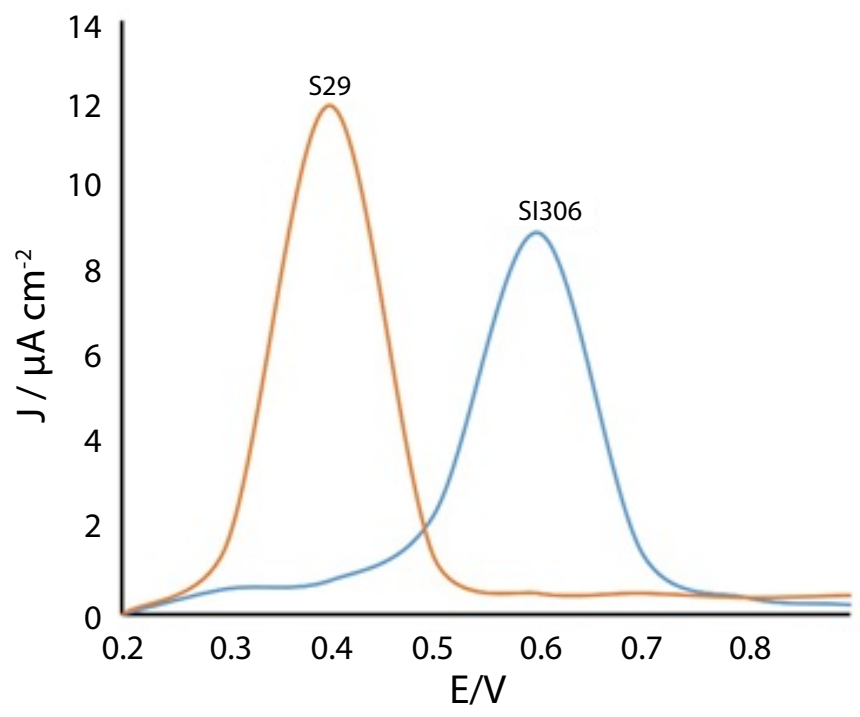

Figure 3. S 29 selectivity study in presence of SI 306, collected by DPV

means that the innovative SPE/GO tools are suitable for in real time pharmacokinetic studies, using disposable, miniaturized and partially automatized electrochemical tools.

Further perspectives and interesting challenges in Medicine, will be represented by the Wireless transmission of the analytical signals (also combined with the IoT_Internet of Things, and ICT_Information Comunication Technologies) and the remote control of big data systems.

\section{References}

1. Li J, Zhang X, Jiang J, Wang Y, Jiang H, et al (2018) Systematic assessment of the toxicity and potential mechanism of graphene derivatives in vitro and in vivo Toxicol Sci. [Crossref] 
Valentini F (2018) Graphene electrochemical sensors for the detection of S 29:1-(2-chloro-2-(4-chlorophenyl)ethyl)-N-(4-fluorobenzyl)-1H-pyrazolo[3,4-d] pyrimidin-4-amine: an anticancer drug

2. Banerjee AN (2018) Graphene and its derivatives as biomedical materials: future prospects and challenges Interface Focus 8: 20170056 [Crossref]

3. Valentini F, Mari E, Zicari A, Calcaterra A, Talamo M, et al (2018) Metal Free Graphene Oxide (GO) Nanosheets and Pristine-Single Wall Carbon Nanotubes (p-SWCNTs) Biocompatibility Investigation: A Comparative Study in Different Human Cell Lines Int J Mol Sci 19: 1316 [Crossref]

4. Valentini F, Calcaterra A, Ruggiero V, Pichichero E, Martino A, et al. (2018) Graphene Oxides synthesised with different antibacterial activities and low cytotoxicity. $J$ Nanomaterials (submitted)

5. Radi M, Brullo C, Crespan E, Tintori C, Musumeci F, et al. (2011) Identification of potent $\mathrm{c}$-Src inhibitors strongly affecting the proliferation of human neuroblastoma cells. Bioorg Med Chem Lett 21: 5928-5933. [Crossref]

6. Ceccherini E, Indovina P, Zamperini C, Dreassi E, Casini N, et al. (2015) SRC Family Kinase Inhibition through a New Pyrazolo [3, 4-d] Pyrimidine Derivative as a Feasible Approach for Glioblastoma Treatment. J Cell Biochem 116: 856-863. [Crossref]
7. Fallacara AL, Mancini A, Zamperini C, Dreassi E, Marianelli S, et al. (2017) Pyrazolo [3, 4-d] pyrimidines-loaded human serum albumin (HSA) nanoparticles: Preparation, characterization and cytotoxicity evaluation against neuroblastoma cell line. Bioorg Med Chem Lett 27: 3196-3200. [Crossref]

8. Manetti F, Brullo C, Magnani M, Mosci F, Chelli B, et al. (2008) StructureBased Optimization of Pyrazolo[3,4-d] pyrimidines as Abl Inhibitors and Antiproliferative Agents toward Human Leukemia Cell Lines. J Med Chem 51: 1252-1259. [Crossref]

9. Martic S, Labib MH, Kraatz B (2011) Electrochemical investigations of sarcomarelated protein kinase inhibition. Electrochimica Acta 56: 10676-10682.

10. Tintori C, Fallacara AL, Radi M, Zamperini C, Dreassi E, et al. (2015) Combining X-ray crystallography and molecular modeling toward the optimization of pyrazolo[3,4-d] pyrimidines as potent c-Src inhibitors active in vivo against neuroblastoma. $J$ Med Chem 58: 347-361. [Crossref]

Copyright: (C2018 Valentini F. This is an open-access article distributed under the terms of the Creative Commons Attribution License, which permits unrestricted use, distribution, and reproduction in any medium, provided the original author and source are credited. 